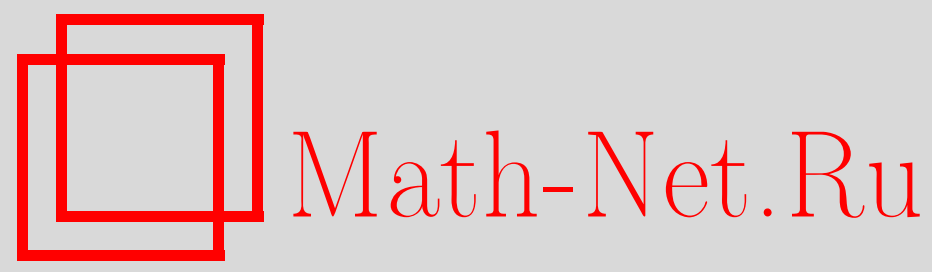

Ш. К. Форманов, А. Н. Старцев, С. С. Седов, Предельные теоремы для обобщённого размера эпидемии в одной марковской модели с иммунизацией, Дискрет. матем., 2013, том 25, выпуск 4, 103-115

DOI: https://doi.org/10.4213/dm1261

Использование Общероссийского математического портала Math-Net.Ru подразумевает, что вы прочитали и согласны с пользовательским соглашением http://www.mathnet.ru/rus/agreement

Параметры загрузки:

IP : 34.239 .49 .27

26 апреля 2023 г., 02:25:03 


\section{Предельные теоремы для обобщённого размера эпидемии в одной марковской модели с иммунизацией*}

() 2013 г. Ш. К. Форманов, А. Н. Старцев, С. С. Седов

В работе предлагается новая марковская модель распространения эпидемии в замкнутой популяции, в которой кроме обычных переходов, отражающих процесс заражения восприимчивых и устранения источников инфекции, вводится также переход, связанный с иммунизацией восприимчивых. Если в известной модели с естественной иммунизацией вероятность последнего перехода пропорциональна числу возможных контактов восприимчивых и больных, то в рассматриваемой модели эта вероятность пропорциональна только числу больных, т.е. с увеличением числа больных вероятность иммунизации возрастает.

Для введенной модели описывается класс предельных законов для обобщенного размера эпидемии (суммы числа зараженных и числа иммунизированных к моменту окончания эпидемии) при различных соотношениях между исходными параметрами модели в предположении, что первоначальные числа восприимчивых и больных стремятся к бесконечности, а параметры модели меняются вместе с ними ("схема серий").

\section{1. Введение}

Введём общую марковскую модель развития эпидемии в замкнутой популяции, с помощью которой мы сможем сопоставить известные ранее модели с моделью, рассматриваемой в данной статье. Пусть $\xi(t)=(R(t), S(t))$ - состояние популяции в данный момент времени $t \geqslant 0$, где $R(t)$ - число восприимчих, а $S(t)$ - число источников инфекции в момент времени $t$, причём $\xi(0)=(n, m)$. Марковскую модель распространения эпидемии зададим с помощью вероятностей возможных переходов за бесконечно малый промежуток времени $\Delta t$

$$
\left\{\begin{array}{l}
\mathbf{P}(\xi(t+\Delta t)=(r-1, s+1) / \xi(t)=(r, s))=\lambda \varphi_{1}(r) \psi(s) \Delta t+o(\Delta t), \\
\mathbf{P}(\xi(t+\Delta t)=(r, s-1) / \xi(t)=(r, s))=\mu \psi(s) \Delta t+o(\Delta t), \\
\mathbf{P}(\xi(t+\Delta t)=(r-1, s) / \xi(t)=(r, s))=\theta \varphi_{2}(r) \psi(s) \Delta t+o(\Delta t),
\end{array}\right.
$$

где первый и второй переходы отражают процесс заражения восприимчивых и устранения больных,соответственно, а третий переход - процесс иммунизации восприимчивых.

\footnotetext{
* Светлой памяти Александра Викторовича Нагаева
} 
Классическая, так называемая общая вероятностная модель, была предложена в 1927 году и связана с именами английских учёных Кермака и МакКендрика [1]. Эта модель получается из (1.1) при $\varphi_{1}(r)=r, \psi(s)=s, \varphi_{2}(r)=0$. Она в дальнейшем была основательно исследована Бартлеттом [2], а сам процесс получил название процесса Бартлетта-МакКендрика (см.,например,[3]).

Более общая модель с $\varphi_{1}(r)=r, \psi(s)=s, \varphi_{2}(r)=r$ была рассмотрена Даунтоном в 1968 г. [4] и независимо от него Нагаевым А.В. и Рахманиной Г.И. в 1970 г. [5], в последней работе третий переход был истолкован как естественная иммунизация, т. е. он отражает возможность перехода восприимчивого в число иммунизированных в результате контакта с источником инфекции.

Для общей вероятностной модели Бартлетт [2] установил уравнение Колмогорова для производящей функции вероятностей состояний процесса

$$
\Pi_{t}(z, w)=\sum_{r=0}^{n} \sum_{s=0}^{m+n-r} \mathbf{P}(\xi(t)=(r, s)) z^{r} w^{s},
$$

которое имеет следующий вид:

$$
\frac{\partial \Pi_{t}(z, w)}{\partial t}=\left(w^{2}-z w\right) \frac{\partial^{2} \Pi_{t}(z, w)}{\partial z \partial w}+\rho_{1}(1-w) \frac{\partial \Pi_{t}(z, w)}{\partial w},
$$

где $\rho_{1}=\mu / \lambda$ - относительный коффициент устранения.

В 1965 году уравнение (1.3) было разрешено в явном виде независимо друг от друга Гани [6] и Сискиндом [7]. Уравнение типа (1.3) с явными коэффициентами при производных оказалось разрешимым лишь благодаря специальному виду неизвестной функции $\Pi_{t}(z, w)$.

$\mathrm{K}$ сожалению, явный вид решения уравнения (1.3) является настолько громоздким, что его трудно использовать для асимптотического анализа или выявления соотношений между исходными параметрами, характерными для того или иного поведения процесса. В связи с этим были предприняты попытки (как до получения явного решения модели, так и после этого) асимптотического анализа распределения размера эпидемии в условиях большой популяции $(n \rightarrow \infty)$. Наиболее общий подход к асимптотическому анализу распределения этого важного функционала был предпринят в работах А.В.Нагаева и его учеников, начиная с 1968 г.([9], [10],[11] и др.). В этих работах был разработан метод сведения задачи о распределении размера эпидемии к граничной задаче для сумм независимых случайных величин в схеме серий.

В настоящей работе проводится аналогичный асимптотический анализ модели $(1.1)$ при $\varphi_{1}(r)=r, \psi(s)=s, \varphi_{2}(r)=1$. В отличие от общей вероятностной модели и модели с естественной иммунизацией здесь возникают специфические трудности реализации граничных задач, а также новые эффекты в соотношениях между исходными параметрами. Вопрос о явном решении уравнения для производящей функции в рассматриваемой модели, а также в модели с естественной иммунизацией, пока остаётся открытым, а задача асимптотического анализа, в силу сказанного выше, остаётся актуальной.

Отметим также, что асимптотический анализ немарковского аналога общей вероятностной модели, в котором учитывается состояние иммунной системы восприимчивых, а инфекционный период имеет произвольное (отличное от показательного) распределение, был рассмотрен в работах [12]-[14]. 


\section{2. Постановка задачи и формулировка результатов}

В работе рассматривается следующая марковская модель развития эпидемии:

$$
\left\{\begin{array}{l}
\mathbf{P}(\xi(t+\Delta t)=(r-1, s+1) / \xi(t)=(r, s))=r s \Delta t+o(\Delta t), \\
\mathbf{P}(\xi(t+\Delta t)=(r, s-1) / \xi(t)=(r, s))=\rho_{1} s \Delta t+o(\Delta t), \\
\mathbf{P}(\xi(t+\Delta t)=(r-1, s) / \xi(t)=(r, s))=\rho_{2} s \Delta t+o(\Delta t),
\end{array}\right.
$$

где $\rho_{1}=\mu / \lambda$ и $\rho_{2}=\theta / \lambda$ - относительные коэффициенты устранения и иммунизации соответственно.

В качестве регулирующих параметров выбираются величины $\theta_{1}=\rho_{1} / n$ и $\theta_{2}=$ $\rho_{2} / n$, в терминах которых и будет проводиться классификация различных видов поведения процесса.

Из определения ясно, что состояния вида $(k, 0), 0 \leqslant k \leqslant n$ являются поглощающими для процесса $\xi(t)$.

Объектом исследования будет обобщённый размер эпидемии $\nu=\nu_{1}+\nu_{2}$, где $\nu_{1}$ и $\nu_{2}$ - числа переболевших и иммунизированных восприимчивых к моменту окончания эпидемии.

Всюду в дальнейшем в данной работе будет предполагаться, что $m \rightarrow \infty, n \rightarrow \infty$, $m=o(n)$, а также что $\lim _{n \rightarrow \infty} \theta_{2}=\theta_{20}, 0 \leqslant \theta_{20}<\infty$. При этом относительно парамера $\theta_{1}$ будут рассматриваться следующие случаи (аналогичные во многих отношениях общей вероятностной модели [10] ):

1) Переходный случай 1-го типа: $\lim _{n \rightarrow \infty} \theta_{1}=\theta_{10}=1$.

2) Регулярный случай: $\theta_{10}>1$ и $\theta_{1}=o(m)$ или $0<\theta_{10}<1$ и $-\infty \leqslant g_{0}<0$, где $g_{0}=\lim _{n \rightarrow \infty} \sqrt{n}\left(\frac{m}{n}+1-\left(\theta_{1}+\theta_{2}\right) \ln \left(1+\frac{1}{\theta_{2}}\right)\right)$.

3) Переходный случай 2 -го типа: $0<\theta_{10}<1$ и $0 \leqslant g_{0} \leqslant \infty$.

4) Переходный случай 3 -го типа: $\theta_{1} \rightarrow \infty, m / \theta_{1} \rightarrow \lambda_{0}, 0 \leqslant \lambda_{0}<\infty$.

Для этих случаев можно дать следующие предварительные комментарии общего характера.

В переходном случае 1-го типа на начальной стадии развития процесса число первоначальных больных будет меняться (при условии, что изменение произошло) на +1 или -1 с вероятностями, близкими к $1 / 2$, т.е. процесс не имеет сноса (тренда) и поглощение происходит за счёт случайных флуктуаций, а сама вероятность поглощения аппроксимируется вероятностью первого выхода винеровского процесса на некоторую гладкую границу. Это происходит при относительно быстром стремлении $\theta_{1}$ к 1 , когда $1-\theta_{1}=O(1 / m)$. При более медленном стремлении, когда $m\left|1-\theta_{1}\right| \rightarrow \infty$, процесс приобретает снос, и картина качественно меняется. В этом случае, а также в регулярном случае, предельная граница в соответствующей граничной задаче вырождается, в связи с этим в качестве предельного выступает нормальное распределение. В переходном случае 3-го типа эпидемия охватывает только конечное число восприимчивых, а в переходном случае второго типа эпидемия охватывает всех восприимчивых с положительной вероятностью. Последняя возможность является существенной особенностью рассматриваемой модели: в модели с $\theta_{2}=0$ такой случай отсутствует.

Формулировку результата начнём с переходного случая первого типа. Символ $\Longrightarrow$ всюду означает слабую сходимость.

Теорема 1. Если $\theta_{1} \rightarrow 1, \beta \equiv m\left(1-\theta_{1}\right) \rightarrow \beta_{0}, \frac{m^{3}}{n} \rightarrow \gamma_{0}<\infty,\left|\beta_{0}\right|<\infty$, mо для 
любого фиксированного $x>0$

$$
\mathbf{P}\left(\nu>\frac{\left(1+\theta_{2}\right) m^{2}}{2} x\right) \Longrightarrow \mathbf{P}\left(w(t)<\frac{1}{\sqrt{x}}+\beta_{0} \sqrt{\frac{x}{2}} t-\frac{\left(1+\theta_{20}\right) \gamma_{0} x^{\frac{3}{2}}}{4 \sqrt{2}} t^{2}, 0 \leqslant t \leqslant 1\right)
$$

где $w(t)$ - стандартный винеровский процесс.

Следствие 1. Если $\gamma_{0}=0$, то в условиях Теоремы 1

$$
\mathbf{P}\left(\nu>\frac{\left(1+\theta_{2}\right) m^{2}}{2} x\right) \Longrightarrow 1-e^{\frac{-\left(\beta_{0}+\left|\beta_{0}\right|\right)}{\sqrt{2\left(1+\theta_{20}\right)}}} \int_{0}^{\beta_{0}^{2} x / 4\left(1+\theta_{20}\right)} p(u) d u
$$

где $p(u)=\frac{\left|\beta_{0}\right| e^{\frac{\left|\beta_{0}\right|}{\sqrt{2\left(1+\theta_{20}\right)}}}}{2 \sqrt{2 \pi\left(1+\theta_{20}\right)}} u^{-3 / 2} e^{-u-\beta_{0}^{2} / 8 u\left(1+\theta_{20}\right)}$.

Следствие 2. Если $к$ тому же $\beta_{0}=0$, то

$$
\mathbf{P}\left(\nu>\left(1+\theta_{2}\right) m^{2} x / 2\right) \Longrightarrow \sqrt{\frac{2}{\pi}} \int_{0}^{1 / \sqrt{x}} e^{-u^{2} / 2} d u .
$$

Теорема 2. Если $\theta_{1} \rightarrow 1$ maк, что $|\beta| \rightarrow \infty, \frac{m^{3}}{n \beta^{2}} \rightarrow \infty$, mo

$$
\mathbf{P}\left(\nu>a_{n}-b_{n} x\right) \Longrightarrow \Phi(x) \equiv \frac{1}{\sqrt{2} \pi} \int_{-\infty}^{x} e^{-u^{2} / 2} d u
$$

где $a_{n}=\sqrt{2 m n}, b_{n}=\sqrt[4]{\frac{2 m^{3}}{n}\left(1+\theta_{20}\right)^{2}}$.

Теорема 3. Если $\theta_{1} \rightarrow 1$ так, что $|\beta| \rightarrow \infty, \frac{m^{3}}{n}=o\left(\beta^{2}\right)$, mo

$$
\mathbf{P}\left(\nu>a_{n}-b_{n} x\right) \Longrightarrow \Phi(x),
$$

$2 \partial e$

$$
\begin{gathered}
a_{n}=\left\{\begin{array}{l}
\frac{2 m^{2}\left(1+\theta_{2}\right)^{2}}{\left(\theta_{1}+\theta_{2}\right) \delta_{1}|\beta|}, \text { если } \beta<0, \\
\frac{n \beta \delta_{1}}{m\left(\theta_{1}+\theta_{2}\right)}, \text { если } \beta>0,
\end{array}\right. \\
b_{n}=\left\{\begin{array}{l}
\frac{n}{m} \sqrt{|\beta| \delta_{1} /\left(1+\frac{n \beta^{2} \delta_{1}}{2 m^{3}\left(1+\theta_{20}\right)}\right), \text { если } \beta<0,} \\
\sqrt{\frac{2 m n\left(1+\theta_{20}\right)^{2}}{\beta \delta_{1}}} /\left(1-\frac{1+\theta_{20}}{\delta_{1}}\right), \text { если } \beta>0,
\end{array}\right.
\end{gathered}
$$

$a \delta_{1}=1+\sqrt{1+\frac{2 m^{3}\left(1+\theta_{20}\right)^{2}}{n \beta^{2}}}$.

Перейдём к описанию регулярного случая.

Теорема 4. Если $\theta_{10}>1$ u $\theta_{1}=o(m)$ или $0<\theta_{10}<1 u-\infty \leqslant g_{0}<0$, mо

$$
\mathbf{P}\left(\nu>a_{n}-b_{n} x\right) \Longrightarrow \Phi(x),
$$


где $a_{n}=\left\{\begin{array}{l}\frac{m\left(1+\theta_{2}\right)}{\theta_{1}-1}, \text { если } \theta_{10}>1, \\ n \alpha_{n}, \text { если } 0<\theta_{10}<1,\end{array}\right.$

$$
b_{n}=\left\{\begin{array}{l}
\frac{\left(1+\theta_{20}\right) \sqrt{m\left(\theta_{10}+\left(\theta_{10}^{2}+\theta_{20}\right)\left(1+\theta_{20}\right)\right)}}{\left(\theta_{10}-1\right)^{3 / 2}}, \text { если } \theta_{10}>1, \\
\frac{\sqrt{n \alpha_{0}\left[1+\theta_{10}^{2} /\left(1+\theta_{20}\right)\left(1+\theta_{20}-\alpha_{0}\right)\right]}\left(1+\theta_{20}-\alpha_{0}\right)}{\alpha_{0}-\left(1-\theta_{10}\right)}, \text { если } \theta_{10}<1,
\end{array}\right.
$$

$\alpha_{n}$ и $\alpha_{0}-$ корни уравнениц $\frac{m}{n}+x+\left(\theta_{1}+\theta_{2}\right) \ln \left(1-\frac{x}{1+\theta_{2}}\right)=0 u x+\left(\theta_{10}+\theta_{20}\right) \ln (1-$ $\left.\frac{x}{1+\theta_{20}}\right)=0$ соответственно, а $g_{0}$ определено на стр. 2.

В переходном случае 2-го типа имеет место следующее утверждение.

Теорема 5. Если $0<\theta_{10}<1 u 0 \leqslant g_{0} \leqslant \infty$, mo

$$
\mathbf{P}(\nu=n) \rightarrow \Phi\left(g_{0} / c_{2}\right),
$$

где

$$
c_{2}^{2}=\frac{\theta_{10}^{2}}{\left(1+\theta_{20}\right) \theta_{20}}+\left(\theta_{10}+\theta_{20}\right) \ln \left(1+\frac{1}{\theta_{20}}\right) .
$$

Последний результат касается переходного случая 3-го типа.

Теорема 6. Если $\theta_{1} \rightarrow \infty, \frac{m}{\theta_{1}} \rightarrow \lambda_{0}, 0<\lambda_{0}<\infty$, mo

$$
\mathbf{P}(\nu=k) \rightarrow \frac{\lambda_{0}^{k}}{k !} e^{-\lambda_{0}}, k=0,1,2, \ldots
$$

\section{3. Редукция к граничной задаче для сумм незави- симых случайных величин}

Общая схема доказательства близка к рассуждениям работ [5] и [10]. Поглощение марковского процесса $(2.1)$ в состоянии $(n-k, 0)$ соответствует обобщённому размеру эпидемии $\nu=k$, и для изучения этого распределения достаточно ограничиться рассмотрением соответствующей вложенной цепи Маркова:

$$
\left\{\begin{array}{l}
\mathbf{P}((r, s) \rightarrow(r-1, s+1))=\frac{r}{n} /\left(\theta_{1}+\theta_{2}+r / n\right), \\
\mathbf{P}((r, s) \rightarrow(r, s-1))=\theta_{1} /\left(\theta_{1}+\theta_{2}+r / n\right), \\
\mathbf{P}((r, s) \rightarrow(r-1, s))=\theta_{2} /\left(\theta_{1}+\theta_{2}+r / n\right) .
\end{array}\right.
$$

Следует отметить, что аналогичное случайное блуждание в общей вероятностной модели было введено Фостером [15].

Для удобства перейдём к эквивалентному случайному блужданию с помощью преобразования $r^{\prime}=n-r, s^{\prime}=n-r-(s-m)$ :

$$
\left\{\begin{array}{l}
P\left(\left(r^{\prime}, s^{\prime}\right) \rightarrow\left(r^{\prime}+1, s^{\prime}\right)\right)=\frac{1-r^{\prime} / n}{1+\theta_{1}+\theta_{2}-r^{\prime} / n}=p_{n r^{\prime}}^{(1)}, \\
P\left(\left(r^{\prime}, s^{\prime}\right) \rightarrow\left(r^{\prime}, s^{\prime}+1\right)\right)=\frac{\theta_{1}}{1+\theta_{1}+\theta_{2}-r^{\prime} / n}=p_{n r^{\prime}}^{(2)}, \\
P\left(\left(r^{\prime}, s^{\prime}\right) \rightarrow\left(r^{\prime}+1, s^{\prime}+1\right)\right)=\frac{\theta_{2}}{1+\theta_{1}+\theta_{2}-r^{\prime} / n}=p_{n r^{\prime}}^{(3)}
\end{array}\right.
$$


Сразу отметим, что здесь и всюду в дальнейшем двойные индексы $n r^{\prime}, n i, n i-1$ и т.д. даются без запятой, что не приведёт к путанице, так как первый индекс $n$ всюду один и тот же и означает номер "серии".

Легко видеть, что блуждание (3.1) начинается в точке $(0,0)$, а поглощающим экраном служат целочисленные точки прямой $s^{\prime}=m+r^{\prime}, r^{\prime}=0, \ldots, n$. Далее, как и в работе [9], (модель(3.1) с $\theta_{2}=0$ ), введём вспомогательные случайные величины (с.в.) $\zeta_{n i}$ как времена пребывания блуждающей частицы на прямой $r^{\prime}=i-1, i=$ $1,2, \ldots, n$. Легко понять, ввиду марковости блуждания (3.1), что эти с.в. независимы и имеют геометрическое распределение

$$
\mathbf{P}\left(\zeta_{n i}=k\right)=\left(1-p_{n i-1}^{(2)}\right)\left(p_{n i-1}^{(2)}\right)^{k}, k=0,1, \ldots, n .
$$

Кроме того, как и в работе [5], введём с.в. $\eta_{n i}$, которые принимают значение 1 , если переход на линию $r^{\prime}=i$ произошёл путём иммунизации восприимчивого, и значение 0, если это осуществилось путём заболевания, т.е.

$$
\eta_{n i}=\left\{\begin{array}{l}
1, \theta_{2} /\left(1+\theta_{2}-\frac{i-1}{n}\right) \\
0, \frac{1-(i-1) / n}{1+\theta_{2}-\frac{i-1}{n}} .
\end{array}\right.
$$

Легко понять, что с.в. $\eta_{n 1}, \eta_{n 2}, \ldots, \eta_{n n-1}$ также будут независимыми. Заметим еще, что в модели с естественной иммунизацией с.в. $\eta_{n i}$ принимают значения 1 и 0 с вероятностями $\theta /(\lambda+\theta)$ и $\lambda /(\lambda+\theta)$ соответственно.

Теперь введём основные с.в.

$$
\xi_{n 1}=\zeta_{n 1}, \xi_{n i}=\zeta_{n i}+\eta_{n i} \text { при } i \geqslant 2 .
$$

Нетрудно установить из (3.2) и (3.3), что

$$
\mathbf{P}\left(\xi_{n i}=k\right)=\left\{\begin{array}{l}
p_{n i-1}^{(1)}, \text { если } k=0, \\
\left(1-p_{n i-1}^{(1)}\right)\left(1-p_{n i-1}^{(2)}\right)\left(p_{n i-1}^{(2)}\right)^{k}, \text { если } k \geqslant 1 .
\end{array}\right.
$$

Далее, введём суммы

$$
S_{n_{K}}=\sum_{i=1}^{k} \xi_{n i}, k=1,2, \ldots n-1, \quad \text { и } S_{n k}^{*}=\frac{S_{n k}-M_{n k}}{B_{n}},
$$

где

$$
M_{n k}=\mathbf{E} S_{n k}, B_{n k}^{2}=\mathbf{D} S_{n k}, B_{n}^{2}=B_{n k_{n}}^{2} .
$$

В терминах этих величин распределение $\nu$ записывается в виде

$$
\mathbf{P}(\nu>k)=\mathbf{P}\left(S_{n 1}<m, S_{n 2}<m+1, \ldots, S_{n k+1}<m+k\right) .
$$

Пусть $\xi_{n}(t)$ - непрерывная случайная ломаная с вершинами в точках $\left(t_{n k}, S_{n k}^{*}\right)$, a $g_{n}(t)$ детерминированная ломаная с вершинами в точках $\left(t_{n k}, \frac{m+k-1-M_{n k}}{B_{n}}\right), k=$ $1,2, \ldots, k_{n}$, где $t_{n k}=\frac{B_{n k}^{2}}{B_{n}^{2}} ;$ тогда $(3.5)$ можно переписать в виде

$$
\mathbf{P}\left(\nu>k_{n}\right)=\mathbf{P}\left(\xi_{n}(t)<g_{n}(t), \quad 0 \leqslant t \leqslant 1\right) .
$$

Соотношение (3.6) и служит основой для получения предельных распределений с использованием принципа инвариантности, согласно которому $f\left(\xi_{n}(t)\right) \Longrightarrow f(w(t))$ для 
любого непрерывного в $[0,1]$ функционала $f$, где $w(t)$ - стандартный винеровский процесс (см., например, [16]). Сразу отметим, что в некоторых из рассматриваемых случаев предельная граница $g(t)$ является непрерывной функцией, а в большинстве других она является вырожденной: $g(1)=$ const, $g(t)=\infty$ при $0<t<1$.

Для обоснования применимости принципа инвариантности необходимо изучить асимптотику моментов и проверить выполнение условий теоремы Линдеберга.

Исходя из (3.2) и (3.3), нетрудно установить, что

$$
\begin{gathered}
m_{n i} \equiv \mathbf{E} \xi_{n i}=\frac{\theta_{1}}{1-\frac{i-1}{n}+\theta_{2}}+\frac{\theta_{2}}{1+\theta_{2}-\frac{i-1}{n}} \\
\sigma_{n i}^{2} \equiv \mathbf{D} \xi_{n i}=\frac{\theta_{1}}{1+\theta_{2}-\frac{i-1}{n}}+\left(\frac{\theta_{1}}{1+\theta_{2}-\frac{i-1}{n}}\right)^{2}+\frac{\theta_{2}\left(1-\frac{i-1}{n}\right)}{\left(1+\theta_{2}-\frac{i-1}{n}\right)^{2}} .
\end{gathered}
$$

Асимптотику величин $M_{n k}$ и $B_{n k}^{2}$ найдём с помощью следующей леммы, доказательство которой не представляет труда.

Лемма 1. Eсли $h(x) \geqslant 0$ и не возрастает на $[0,1]$, то для любых $\alpha \in(0,1]$ u $k_{n}<n$

$$
\sum_{k=0}^{k_{n}} h\left(1-\alpha \frac{k}{n}\right)=\frac{n}{\alpha} \int_{1-\frac{\alpha k_{n}}{n}}^{1} h(x) d x+R_{n},
$$

где $h(1) \leqslant R_{n} \leqslant h\left(1-\alpha \frac{k_{n}}{n}\right)$.

Отсюда нетрудно получить, что если $k \leqslant n(1-\delta), 0<\delta<1$, то

$$
M_{n k+1}=-n\left(\theta_{1}+\theta_{2}\right) \ln \left(1-\frac{k}{n\left(1+\theta_{2}\right)}\right)+\frac{\theta_{1}+\theta_{2}}{1+\theta_{2}} R_{n_{1}},
$$

$B_{n k+1}^{2}=\frac{k \theta_{1}^{2}}{\left(1+\theta_{2}\right)^{2}} \frac{1}{1-\frac{k}{\left(1+\theta_{2}\right) n}}-n\left(\theta_{1}+\theta_{2}\right) \ln \left(1-\frac{k}{n\left(1+\theta_{2}\right)}\right)+\frac{\theta_{1}+\theta_{2}}{1+\theta_{2}} R_{n_{1}}+\frac{\theta_{1}^{2}-\theta_{2}^{2}}{\left(1+\theta_{2}\right)^{2}} R_{n_{2}}$,

где $1 \leqslant R_{n_{1}} \leqslant \frac{1+\theta_{2}}{\theta_{2}+\delta}, 1 \leqslant R_{n_{2}} \leqslant \frac{\left(1+\theta_{2}\right)^{2}}{\left(\theta_{2}+\delta\right)^{2}}$.

Аналогичным образом можно оценить и сумму третьих моментов. С помощью этих результатов нетрудно оценить дробь Ляпунова. Для $k_{n} \rightarrow \infty$ и $k_{n} \leqslant(1-\delta) n$ :

$$
L_{3 n} \equiv \frac{1}{B_{n}^{3}} \sum_{i=1}^{k_{n}} \mathbf{M}\left|\xi_{n_{i}}-m_{n_{i}}\right|^{3}=O\left(\frac{1}{\sqrt{k_{n}}}\right) .
$$

Заметим также, что при $k \rightarrow \infty$ и $k=o(n):$

$$
\begin{gathered}
M_{n k+1}=\frac{\theta_{1}+\theta_{2}}{1+\theta_{2}} k+\frac{\theta_{1}+\theta_{2}}{2\left(1+\theta_{2}\right)^{2}} \frac{k^{2}}{n}+O\left(\frac{k^{3}}{n^{2}}\right), \\
B_{n k+1}^{2} \sim \sigma^{2}(n) k, \sigma^{2}(n)=\left(\frac{\theta_{1}}{1+\theta_{2}}\right)^{2}+\frac{\theta_{1}}{1+\theta_{2}}+\frac{\theta_{2}}{\left(1+\theta_{2}\right)^{2}} .
\end{gathered}
$$




\section{4. Доказательство основных утверждений}

Доказательство теоремь 1. В условиях этой теоремы $k_{n}=o(n)$. Поэтому из $(3.9)$ и $(3.10)$ имеем, что $t_{n k} \sim \frac{k}{k_{n}}$ и при этом

$$
g_{n}\left(t_{n k}\right)=\frac{m+k-\frac{\theta_{1}+\theta_{2}}{1+\theta_{2}} k-\frac{\theta_{1}+\theta_{2}}{\left(1+\theta_{2}\right)^{2}} \frac{k^{2}}{n}+O\left(k^{3} / n^{2}\right)}{\sigma(n) \sqrt{k_{n}}} .
$$

Выбирая $k_{n}=\left(1+\theta_{2}\right) m^{2} x / 2$ и замечая, что $\sigma^{2}(n) \sim \frac{2}{1+\theta_{20}}$, имеем

$$
\begin{gathered}
g_{n}\left(t_{n k}\right)=\frac{1}{\sqrt{x}}+\left(1-\frac{\theta_{1}+\theta_{2}}{1+\theta_{2}}\right) \frac{k}{k_{n}} \sqrt{k_{n}}-\frac{\theta_{1}+\theta_{2}}{2\left(1+\theta_{2}\right)^{2}}\left(\frac{k}{k_{n}}\right)^{2} \frac{k_{n}^{3 / 2}}{n}+O\left(\frac{k_{n}}{n^{2}}\right)^{5 / 2}= \\
=\frac{1}{\sqrt{x}}+\frac{\left(1-\theta_{1}\right) m \sqrt{x}}{\sqrt{2} \sqrt{1+\theta_{2}}} t_{n k}-\frac{\theta_{1}+\theta_{2}}{\sqrt{1+\theta_{2}}} \frac{m^{3} x^{3 / 2}}{n \cdot 2^{3 / 2}} t_{n k}^{2}+O\left(\frac{1}{m}\right) .
\end{gathered}
$$

Теперь, если $k \sim k_{n} t, 0<t<1$, то

$$
g(t)=\lim _{n \rightarrow \infty} g_{n}\left(t_{n k}\right)=\frac{1}{\sqrt{x}}+\frac{\beta_{0} \sqrt{x / 2}}{\sqrt{1+\theta_{20}}} t-\frac{\left(\theta_{10}+\theta_{20}\right) \gamma_{0}(x / 2)^{3 / 2}}{2 \sqrt{\left(1+\theta_{20}\right)}} t^{2} .
$$

Таким образом, в силу принципа инвариантности

$$
\mathbf{P}\left(\nu_{n}>k_{n}\right) \Longrightarrow \mathbf{P}(w(t)<g(t), 0 \leqslant t \leqslant 1) .
$$

Утверждение следствия 1 следует из явного представления

$$
\mathbf{P}(w(t)<b+k t, 0 \leqslant t \leqslant T)=1-e^{-b(k+|k|)} \int_{0}^{T k^{2} / 2} p_{b, k}(u) d u,
$$

где $p_{b, k}(u)=\frac{b|k| e^{b|k|}}{2 \sqrt{\pi}} u^{-3 / 2} e^{-u-b^{2} k^{2} / 4 u}-$ плотность распределения Вальда. Это равенство легко выводится из следующей формулы, приведённой в монографии Такача $[17, \mathrm{c} 92]$ :

$$
\mathbf{P}(w(t)<b+k t, 0 \leqslant t \leqslant T)=1-b \int_{0}^{T} \varphi\left(\frac{b+k t}{\sqrt{t}}\right) t^{-3 / 2} d t,
$$

где $\varphi(x)$ - плотность стандартного нормального распределения.

Доказательство теорем 2,3 и 4. Эти теоремы относятся к случаю вырожденной предельной границы, т.е. когда

$$
g(t)=\infty \text { при } t \in(0,1), g(1)=x=\text { const },
$$

и тогда

$$
\mathbf{P}\left(\nu>k_{n}\right) \Longrightarrow \mathbf{P}(w(1)<x)=\Phi(x) .
$$

Как и в случае общей вероятностной модели $\left(\theta_{2}=0\right)$, эти теоремы доказываются схожим образом . Последовательность $k_{n}$ выбирается так, что $k_{n}=n \alpha_{n}-b_{n} x$, где $\alpha_{n}$ - положительный корень уравнения

$$
f_{n}(x) \equiv \frac{m}{n}+x+\left(\theta_{1}+\theta_{2}\right) \ln \left(1-\frac{x}{1+\theta_{2}}\right)=0 .
$$


В терминах $f_{n}(x)$ искомая вероятность запишется следующим образом:

$$
\mathbf{P}\left(\nu>k_{n}\right)=\mathbf{P}\left(S_{n k}^{*}<\frac{n f_{n}\left(\frac{k}{n}\right)}{B_{n}}+o(1), 1 \leqslant k \leqslant k_{n}\right) .
$$

При этом

$$
g_{n}(1)=\frac{n}{B_{n}} f_{n}\left(\frac{k_{n}}{n}\right) \sim \frac{f_{n}^{\prime}\left(\alpha_{n}\right)}{B_{n}}\left(k_{n}-n \alpha_{n}\right) \sim \frac{f_{n}^{\prime}\left(\alpha_{n}\right)}{B_{n}} b_{n} \cdot x \sim x,
$$

если $b_{n} \sim B_{n} / f_{n}^{\prime}\left(\alpha_{n}\right)$.

Вырожденность предельной границы доказывается путём выбора таких последовательностей $t_{n}^{\prime} \rightarrow 1$ и $t_{n}^{\prime \prime} \rightarrow 0$, что $\lim _{n \rightarrow \infty} g_{n}\left(t_{n}^{\prime}\right)=\lim _{n \rightarrow \infty} g_{n}\left(t_{n}^{\prime \prime}\right)=\infty$. Выбор таких последовательностей в каждом случае не представляет особого труда. Например, как и в [10], в качестве $t_{n}^{\prime \prime}$ можно взять $t_{n k_{n}^{\prime}}=1-B_{k_{n}^{\prime}}^{2} / B_{n}^{2}$ где $k_{n}^{\prime}=a_{n}-b_{n} x_{n}$ с $x_{n} \rightarrow \infty$ и $x_{n}=o\left(a_{n} / b_{n}\right)$, так как в рассматриваемых теоремах $b_{n}=o\left(a_{n}\right)$. $\mathrm{A}$ в качестве $t_{n}^{\prime}$ можно выбрать какое-то из $t_{n i}$ с $i \rightarrow \infty$ с нужными свойствами.

Доказательство теоремы 2. В условиях этой теоремы корень уравнения (4.2) стремится к нулю, и для нахождения его асимптотики достаточно рассмотреть квадратичное приближение к функции $f_{n}(x)$, т. е. решить уравнение

$$
\frac{\theta_{1}+\theta_{2}}{\left(1+\theta_{2}\right)^{2}} x^{2}-2 \frac{1-\theta_{1}}{1+\theta_{2}} x-2 \frac{m}{n}=0 .
$$

Следовательно, в этом случае имеем

$$
\alpha_{n}=\left(1-\theta_{1}\right)+\sqrt{\left(1-\theta_{1}\right)^{2}+2 \frac{m}{n}\left(\theta_{1}+\theta_{2}\right)} \frac{1+\theta_{2}}{\theta_{1}+\theta_{2}} .
$$

А так как $1-\theta_{1}=\frac{\beta}{m}=o\left(\sqrt{\frac{m}{n}}\right)$ в силу условия $\frac{n}{m^{3}}=o\left(\frac{1}{\beta^{2}}\right)$, то $\alpha_{n} \sim \sqrt{2\left(1+\theta_{20}\right) \frac{m}{n}}$.

Выбор нормирующей последовательности $b_{n}$, в соответствии с (4.4), осуществляется так, чтобы $b_{n} \sim-\frac{B_{n}}{f_{n}^{\prime}\left(\alpha_{n}\right)}$, где

$$
B_{n} \sim \sqrt{\frac{2 k_{n}}{1+\theta_{2}}} \sim \sqrt{\frac{2 n \alpha_{n}}{1+\theta_{2}}}
$$

Следовательно, $b_{n} \sim \sqrt{\frac{2 n\left(1+\theta_{20}\right)}{\alpha_{n}}}$, так как $1-\theta_{1}=o\left(\alpha_{n}\right)$.

Далее, в условиях данной теоремы имеем

$$
g_{n}\left(t_{n 1}\right)=\frac{m}{B_{n}} \sim \frac{m \sqrt{1+\theta_{20}}}{\sqrt{2 \sqrt{n} \sqrt{2\left(1+\theta_{20}\right) \frac{m}{n}}}}=\frac{m^{\frac{3}{4}}\left(1+\theta_{20}\right)^{\frac{3}{4}}}{2^{\frac{3}{4}} n^{\frac{1}{4}}}=\left(\frac{1+\theta_{20}}{2}\right)^{\frac{3}{4}}\left(\frac{m^{3}}{n}\right)^{\frac{1}{4}} \rightarrow \infty .
$$

Тем самым теорема доказана.

Доказательство теоремы 3. Если $\beta<0$ (т.е. $\theta_{1}>1$ ), то

$$
\alpha_{n}=\left[\sqrt{\left(\frac{1-\theta_{1}}{1+\theta_{2}}\right)^{2}+2 \frac{m}{n}}-\frac{\theta_{1}-1}{1+\theta_{2}}\right] \cdot \frac{1+\theta_{2}}{\theta_{1}+\theta_{2}}=\frac{2 m^{2}\left(1+\theta_{2}\right)^{2}}{n|\beta|\left(\theta_{1}+\theta_{2}\right) \delta_{1}} \sim \frac{2 m^{2}\left(1+\theta_{2}\right)}{n|\beta| \delta_{1}},
$$


где

$$
\delta_{1}=1+\sqrt{1+2 \frac{m^{3}\left(1+\theta_{2}\right)^{2}}{n \beta^{2}}} .
$$

В этом случае

$$
g_{n}\left(t_{n 1}\right)=\frac{m}{B_{n}} \sim \frac{m \sqrt{1+\theta_{2}}}{\sqrt{2 k_{n}}} \sim \frac{m \sqrt{1+\theta_{2}} \sqrt{n|\beta| \delta_{1}}}{\sqrt{2 n 2 m^{2}\left(1+\theta_{20}\right)}}=\frac{1}{2} \sqrt{\delta_{1}|\beta|} \rightarrow \infty .
$$

Если же $\beta>0$ ( т.е. $\left.\theta_{1}<1\right)$, то корень $\alpha_{n}$ имеет тот же вид, что и в (4.6). После преобразований получим

$$
\alpha_{n}=\frac{1-\theta_{1}}{\theta_{1}+\theta_{2}}\left[1+\sqrt{1+2 \frac{m\left(1+\theta_{2}\right)^{2}}{n\left(1-\theta_{1}\right)^{2}}}\right]=\frac{\beta \delta_{1}}{m\left(\theta_{1}+\theta_{2}\right)} .
$$

Но в рассматриваемом случае, как нетрудно убедиться, $y_{n}\left(t_{n 1}\right)=O\left(\frac{m^{3}}{n \beta^{2}}\right)=$ $O(1)$. Однако, если выбрать $t_{n}^{\prime \prime}=\frac{B_{n i}^{2}}{B_{n}^{2}}$ с $i=\left[\frac{n \beta}{m}\right]^{3 / 4}$, то $t_{n}^{\prime \prime} \rightarrow 0$, a $g_{n}\left(t_{n}^{\prime \prime}\right) \rightarrow \infty$.

Доказательство теоремы 4. 1. Пусть сначала $\theta_{10}>1$ и $\theta_{1}=o(m)$. В этом случае корень уравнения $f_{n}(x)=0$ также стремится к нулю. Применяя квадратичное приближение, из уравнения (4.5), с учётом того, что $m=o(n)$, имеем

$$
\begin{gathered}
\alpha_{n}=\left(\sqrt{\left(\frac{1-\theta_{1}}{1+\theta_{2}}\right)^{2}+2 \frac{m}{n}}-\frac{\theta_{1}-1}{1+\theta_{2}}\right) \cdot \frac{1+\theta_{2}}{\theta_{1}+\theta_{2}}= \\
\frac{2 m / n}{\sqrt{\left(\frac{1-\theta_{1}}{1+\theta_{2}}\right)^{2}+2 \frac{m}{n}}+\frac{\theta_{1}-1}{1+\theta_{2}}} \cdot \frac{1+\theta_{2}}{\theta_{1}+\theta_{2}}=\frac{m\left(\theta_{1}+\theta_{2}\right)}{n\left(\theta_{1}-1\right)}\left(1+O\left(\frac{m}{n}\right)\right) .
\end{gathered}
$$

В рассматриваемом случае $B_{n}^{2} \sim n \alpha_{n} \sigma^{2}(n) \sim m c_{0}^{2}$, где

$$
c_{0}^{2}=\frac{\left(\theta_{10}+\theta_{20}\right)}{\theta_{10}-1} \cdot\left(\frac{\theta_{10}}{1+\theta_{20}}+\frac{\theta_{10}^{2}}{\left(1+\theta_{20}\right)^{2}}+\frac{\theta_{20}}{\left(1+\theta_{20}\right)^{2}}\right) .
$$

В силу этого

$$
g_{n}\left(t_{n 1}\right)=\frac{m}{B_{n}} \sim \frac{\sqrt{m}}{c_{0}} \rightarrow \infty .
$$

Величина $b_{n}$ при этом принимает вид $b_{n}=\delta_{0} \sqrt{m}$, где

$$
\delta_{0}=\frac{\left(1+\theta_{20}\right)}{\left(\theta_{10}-1\right)^{3 / 2}}+\sqrt{\theta_{10}+\frac{\theta_{20}+\theta_{10}^{2}}{1+\theta_{20}}} .
$$

2. Если $0<\theta_{10}<1$ и при этом выполняется условие $-\infty \leqslant g_{0}<0$, то уравнение $f_{n}(x)=0$ имеет положительный корень $\alpha_{n}, 1-\theta_{1}<\alpha_{n} \leqslant 1$, причём $\alpha_{n} \rightarrow \alpha_{0}$, где $\alpha_{0}$ - корень уравнения

$$
x+\left(\theta_{10}+\theta_{20}\right) \ln \left(1-\frac{x}{1+\theta_{20}}\right)=0 .
$$


В условиях рассматриваемого случая, в силу (3.8), имеем

$$
B_{n}^{2} \sim \frac{n \alpha_{0} \theta_{10}^{2}}{\left(1+\theta_{20}\right)^{2}} \cdot \frac{1}{1-\frac{\alpha_{0}}{1+\theta_{20}}}-n\left(\theta_{10}+\theta_{20}\right) \ln \left(1-\frac{\alpha_{0}}{1+\theta_{20}}\right) .
$$

А так как $\left(\theta_{10}+\theta_{20}\right) \ln \left(1-\frac{\alpha_{0}}{1+\theta_{20}}\right)=-\alpha_{0}$, то

$$
B_{n}^{2} \sim n \alpha_{0}\left(1+\theta_{10}^{2} /\left(1+\theta_{20}\right)\left(1+\theta_{20}-\alpha_{0}\right)\right) \equiv n c_{1}^{2} .
$$

В силу этого $g_{n}\left(t_{n 1}\right)=\frac{m}{B_{n}} \sim \frac{m}{c_{1} \sqrt{n}}$, и поэтому в качестве $t_{n}^{\prime \prime}$ выберем $t_{n i}$ с $i=\left[n^{5 / 8}\right]$, для которого будет выполнено необходимое условие.

Тем самым теорема 4 доказана.

Что касается теоремы 5, то её утверждение очевидно ввиду того, что при её условиях $B_{n}^{2} \sim n c_{2}^{2}$, где $c_{2}^{2}=\frac{\theta_{10}^{2}}{\left(1+\theta_{20}\right) \theta_{20}}+\left(\theta_{10}+\theta_{20}\right) \ln \left(1+\frac{1}{\theta_{20}}\right)$, и, следовательно,

$$
g_{n}(1) \sim \frac{n f_{n}(1)}{B_{n}} \sim \frac{\sqrt{n} f_{n}(1)}{C_{2}} \sim \frac{g_{0}}{C_{2}} .
$$

Доказательство теоремы 6. Легко понять, что в условиях рассматриваемой теоремы $p_{n i}^{(2)} \rightarrow 1$ и $p_{n i}^{(2)} \sim p_{n 0}^{(2)}=\frac{\theta_{1}}{1+\theta_{1}+\theta_{2}}$ при любом фиксированном $i$.

Следовательно, вместо с.в. $\xi_{n i}$ можно рассматривать (при $i \geqslant 2$ ) такие $\widetilde{\xi_{n i}}$, что

$$
\mathbf{P}\left(\widetilde{\xi_{n_{i}}}=k\right)=\left\{\begin{array}{l}
p_{n_{0}}^{(1)}, \quad \text { если } k=0 \\
\left(1-p_{n_{0}}^{(1)}\right)\left(1-p_{n_{0}}^{(2)}\right)\left(p_{n_{0}}^{(2)}\right)^{k}, \text { если } k \geqslant 1,
\end{array}\right.
$$

а $\widetilde{\xi}_{n 1}$ имеет распределение $(3.2)$ с $p_{n i-1}^{(2)}=p_{n 0}^{(2)}$. Тогда

$$
\begin{gathered}
\mathbf{P}(\nu=k) \sim \mathbf{P}\left(\widetilde{\xi}_{n 1}<m, \widetilde{\xi}_{n 1}+\widetilde{\xi}_{n 2}<m+1, \ldots, \widetilde{\xi}_{n 1}+\ldots+\widetilde{\xi}_{n n k+1} \geqslant m+k\right)= \\
=\sum_{i_{1}<m} \mathbf{P}\left(\widetilde{\xi}_{n 1}=i_{1}\right) \sum_{i_{2}<m+1-i_{1}} \mathbf{P}\left(\widetilde{\xi}_{2}=i_{2}\right) \ldots \sum_{i_{k}<m+k-1-i_{1}-\ldots-i_{k-1}} \mathbf{P}\left(\widetilde{\xi}_{n k}=i_{k}\right) \\
\sum_{i_{k+1} \geqslant m+k-i_{1}-\ldots-i_{k}} \mathbf{P}\left(\widetilde{\xi}_{n k+1}=i_{k+1}\right) .
\end{gathered}
$$

Далее, легко заметить, что суммирование по $i_{2}, \ldots, i_{k-1}$ в $(4.8)$ можно ограничить значениями $i_{2} \geqslant 1, \ldots, i_{k} \geqslant 1$, так как в силу $(4.7)$ значения $i_{2}=0, \ldots, i_{k}=0$ влекут за собой появление слагаемых, содержащих $p_{n 0}^{(1)}$, которые стремятся к нулю при $n \rightarrow \infty$, поэтому, с учётом (4.7), имеем

$$
\mathbf{P}(\nu=k)=\left(1-p_{n 0}^{(1)}\right)^{k}\left(1-p_{n 0}^{(2)}\right)^{k}\left(p_{n 0}^{(2)}\right)^{m} \cdot N_{k}(m)+o(1),
$$

где $N_{k}(m)=\left|D_{k}(m)\right|$ - число точек в области

$$
D_{k}(m)=\left\{\left(\alpha_{1}, \ldots \alpha_{k}\right): i_{1}<m, \ldots, i_{1}+\ldots+i_{k}<m+k-1, i_{2} \geqslant 1, \ldots, i_{k} \geqslant 1\right\} .
$$

Легко установить, что величина $N_{k}(m)$ удолетворяет рекуррентному соотношению

$$
N_{k}(m)=\sum_{i=1}^{m} N_{k-1}(i)
$$


Основную идею доказательства продемонстрируем для $k=2$. Если $D_{1}(m)=$ $\left\{i_{1}: i_{1}<m\right\}$, то

$$
\begin{gathered}
D_{2}(m)=\left\{\left(i_{1}, i_{2}\right): i_{1}<m, i_{1}+i_{2}<m+1 ; i_{2} \geqslant 1\right\}= \\
=\cup_{i=1}^{m}\left\{\left(i_{1}, i_{2}\right): i_{1}<m, i_{1}+i_{2}<m+1 ; i_{2}=i\right\}= \\
=\cup_{i=1}^{m}\left\{i_{1}: i_{1}<m+1-i\right\}=\cup_{j=1}^{m} D_{1}(j) .
\end{gathered}
$$

Далее, нетрудно показать, что $N_{k}(m)=C_{m+k-1}^{k}$. Это легко обосновывается индукцией по $m$ с использованием соотношения (4.10) и тождества $\sum_{k=1}^{m} C_{k+a}^{k-1}=$ $C_{m+a+1}^{m-1}$. Подставляя $N_{k}(m)$ в соотношение $(4.9)$, получим

$$
\begin{aligned}
& \mathbf{P}(\nu=k) \sim\left(1-p_{n 0}^{(1)}\right)^{k}\left(1-p_{n 0}^{(2)}\right)^{k}\left(p_{n 0}^{(2)}\right)^{m} \frac{(m+k-1) !}{k !(m-1) !} \sim \\
& \left.\sim \frac{\left(m\left(1-p_{n 0}^{(2)}\right)\right)^{k}}{k !}\left(1-\frac{m\left(1-p_{n 0}^{(2)}\right)}{m}\right)\right)^{m} \sim \frac{\lambda_{0}^{k}}{k !} e^{-\lambda_{0}},
\end{aligned}
$$

что и требовалось доказать.

\section{Список литературы}

1. Kermack W. O., McKendrick A. G., Contributions to the mathematical theory of epidemics. Proc.Roy.Lond.Soc. (1927) A 115, 700-721.

2. Бартлетт М. С., Ввведение в теорию случайных процессов. ИЛ, Москва, 1958.

3. Баруча-Рид А. Т., Элементы теории марковских процессов и их приложения. "Наука", Москва, 1969.

4. Downton F., The ultimate size of carrier-born epidemic. Biometrika (1968) 55, №2, 277289.

5. Нагаев А. В., Рахманина Г. И., Пороговые теоремы для стохастической модели с иммунизацией. Мат.заметки (1970) 3, №3, 385-392.

6. Gani J., On the partial differential equation of epidemic theory I. Biometrika (1965) 52, №3-4, 617-622.

7. Siskind. V., A solution of general stochastic epidemic. Biometrika (1965) 52, №3-4, 613616.

8. Bailey N. T. J., The mathematical theory of epidemic, London, 1957.

9. Нагаев А. В.,Старцев А. Н., Пороговая теорема для одной модели эпидемии. Мат. заметки. (1968) 3, №2, 179-185.

10. Нагаев А. В.,Старцев А. Н., Асимптотический анализ одной стохастической модели эпидемии. Теория вероятностей и её прим. (1970) XV, №1, 97-105.

11. Нагаев А. В., Некоторые предельные теоремы для общей стохастической модели эпидемии. Матем. заметки (1973) 13, №5, 709-714.

12. Старцев А. Н., Об одной модели с взаимодействием частиц двух типов, обобщающей процесс эпидемии Бартлетта-МакКендрика. Теория вероятностей и её применения (2001) 46, №3, 463-482.

13. Startsev A. N., Asymptotic analysis of the general stochastic epidemic with variable infections periods. J. Aplied Probablity (2001) 38, 18-35. 
14. Мирзаев М.,Старцев А. Н., Предельные теоремы для одной модели с взаимодействием частиц двух типов, обобщающей процесс эпидемии Бартлетта-МакКендрика. Теория вероятностей и её применения (2006) 51, №2, 385-390.

15. Foster F.G., Note on Bailey's and Whitte's treatment of a general stochastic epidemic. Biometrika (1955) 42, 123-125.

16. Гихман И. И., Скороход А. В., Введение в теорию случайных процессов. Наука, 1965.

17. Такач Л., Комбинаторные методы в теории случайных процессов. Мир, Москва, 1974.

Статья поступила 27.01.2012.

Переработанный вариант поступил 02.07.2013. 